\title{
Relativistically smeared X-ray reprocessed components in the GINGA spectra of GS $2023+338$
}

\author{
Piotr T. Życki and Chris Done \\ Department of Physics, University of Durham, South Road, Durham DH1 3LE, England; \\ piotr.zycki@durham.ac.uk, chris.done@durham.ac.uk \\ and \\ David A. Smith \\ Department of Physics and Astronomy, University of Leicester, University Road, Leicester LE1 \\ 7RH, England; das@star.le.ac.uk
}

\begin{abstract}
We present results of spectral analysis of Ginga data obtained during the decline phase after the 1989 outburst of GS 2023+338 (V404 Cyg). Our analysis includes detailed modelling of the effects of X-ray reflection/reprocessing. We have found that (1) the contribution of the reprocessed component (both continuum and line) corresponds to the solid angle of the reprocessor as seen from the X-ray source of $\Omega \approx(0.4-0.5) \times 2 \pi$, (2) the reprocessed component (both line and continuum) is broadened ("smeared") by kinematic and relativistic effects, as expected from the accretion disk reflection. We discuss the constraints these results give on various possible system geometries.
\end{abstract}

Subject headings: accretion, accretion disk - black hole physics - binaries: close - stars: individual (V404 Cyg) - X-ray: stars

\section{Introduction}

Some of the strongest evidence for the existence of accretion disks around black holes has come from X-ray observations of the relativistically smeared iron $\mathrm{K} \alpha$ line profile in Active Galactic Nuclei (Tanaka et al. 1995; Iwasawa et al. 1996; Nandra et al. 1997). This fluorescence line is produced by hard X-ray illumination of the accreting material, and the combination of high orbital velocities and strong gravity in the vicinity of a black hole gives the line a characteristically skewed, broad profile (Fabian et al. 1989; Laor et al. 1991). A reflected continuum should also accompany this line (e.g. Lightman \& White 1988; George \& Fabian 1991; Matt, Perola \& Piro 1991), and the amplitude of both reprocessed components gives constraints on the solid angle subtended by the accreting material, its inclination, elemental abundance and ionization state. Both the amount of reflection/fluorescence and the intensity of the relativistic effects on the observed line 
profile strongly support the idea that the accretion disk extends down to the last stable orbit in AGN. The Black Hole Candidates (BHC) show many X-ray spectral similarities to AGN, plausibly because both involve the same physical processes of disk accretion onto a black hole (Inoue 1993; Ueda, Ebisawa \& Done 1994; Gierliński et al. 1997).

Many Soft X-ray Transients (SXT) are known to be BHC. Their mass accretion rate varies over at least 2 orders of magnitude from outburst (where it is at about Eddington) through the decline on time-scales of months (see Tanaka \& Shibazaki 1996 for recent review). A number of these systems were observed by Ginga and the spectra obtained are amongst the best in which to investigate the overall effects of $\mathrm{X}$-ray reprocessing. Moderate spectral resolution ( $18 \%$ at $6 \mathrm{keV}$ ) is more than compensated by very high signal-to-noise, broad band pass (from $1 \mathrm{keV}$ up to 20-30 $\mathrm{keV}$ ) and our relatively good understanding of the instrument.

In this Letter we present results of spectral analysis of data obtained during the 1989 outburst of GS 2023+338 (V404 Cyg; Kitamoto et al. 1989). The outburst was well covered by Ginga from its initial detection by All Sky Monitor on May 22 until November 1989 (Tanaka \& Lewin 1995). We have selected two data sets where there is little short time-scale spectral variability, obtained June 20 and July 19-20, respectively. We analyse these using models of X-ray reprocessing that consistently connect the properties of the iron $\mathrm{K} \alpha$ line with the properties of the reflected continuum. We show that the reflected component is present in the spectra, that it is smeared by kinematic and relativistic effects as expected from an accretion disk, and that both its normalization and amount of smearing imply that the disk does not extend down to the last stable orbit.

A full analysis of the data covering the outburst and entire decline phase will be presented in future paper (்̇ycki et al., in preparation, hereafter paper II).

\section{Data selection and reduction}

The data were extracted from the original FRF's using the Ginga reduction software at Leicester University. Paradoxically, background subtraction poses a problem for a source as bright as GS $2023+338$ as the background monitors are strongly contaminated by source counts. However, the background can be estimated from nearby observations at similar points in the satellite orbit, and its fractional contribution is low $(\leq 3 \%$ below $10 \mathrm{keV})$. Full details of our method of background subtraction will be given in paper II. We allow for $0.5 \%$ systematic error in the data.

\section{Model}

The model components are: multi-temperature accretion disk spectrum ("disk black body"; Mitsuda et al. 1984) and a power law with its Compton reflection from a possibly ionized medium including the iron $\mathrm{K} \alpha$ line emission. The Compton reflected component is computed using the 
XSPEC version 9.01 model "pexriv" (Magdziarz \& Zdziarski 1995) with the ionization parameterised by the ionization parameter, $\xi \equiv L_{\mathrm{X}} / n r^{2}$ as in Done et al. (1992). We assume elemental abundances of Morrison \& McCammon (1983) with the exception of the iron abundance, [Fe], which is a free parameter. The iron $\mathrm{K} \alpha$ line is computed using modified Monte Carlo simulations code of Życki \& Czerny (1994). We updated the Fe K $\alpha$ line energies, fluorescent yields and Fe K-shell edge energies after Kaastra \& Mewe (1993).

The reprocessed component can then be "smeared" to simulate the relativistic and kinematic effects of disk emission (see e.g. Fabian et al. 1989; Ross, Fabian \& Brandt 1996). We assume a non-rotating black hole so the model is parameterised by the inner and outer radius of the disk, $R_{\text {in }}$ and $R_{\text {out }}$ respectively, and the radial distribution of irradiation emissivity. We fix the form of the emissivity, $F_{\text {irr }}(r) \propto r^{-3}$ as expected from coronal illumination, fix $R_{\text {out }}$ at $10^{4} R_{\mathrm{g}}$, since it is an appropriate value for LMXRB $\left(R_{\mathrm{g}} \equiv G M / c^{2}\right)$ and fit $R_{\text {in }}$ and the normalization of the reprocessed component, $f \equiv \Omega / 2 \pi$, where $\Omega$ is the solid angle of the reprocessor as seen from the X-ray source. We emphasize that $R_{\text {in }}, R_{\text {out }}$ and $F_{\text {irr }}(r)$ describe only the smearing effect, not $\Omega$.

We assume that the inclination of the system is $i=56^{\circ}$ (Pavlenko et al. 1996).

\section{Results of model fitting}

We begin with the simplest possibility, that is a power law spectrum with a narrow line at 6.4 $\mathrm{keV}$. We than add the reflected continuum assuming first that it is un-ionized, non-smeared and the iron abundance is 1 relative to cosmic value. In the next step we fit $\xi$ and $[\mathrm{Fe}]$ and finally we introduce the effect of smearing. This multi-step procedure is important (1) to demonstrate the significance of reprocessing and (2) since the moderate spectral resolution of Ginga means that the broadening of iron spectral features due to ionization can mimic the relativistic smearing.

For the June 20th data set, the simplest model (model 0 in Table 1 ) gives $\chi_{\nu}^{2}=1.9$ and it is thus not acceptable. Adding the reprocessed component is highly significant even in the simplest version (model A), $\chi^{2}=19.9 / 25$ d.o.f. $\left(\chi_{\nu}^{2}=0.80\right)$. We note that the normalization of the reflected component is significantly smaller than $1, f=0.46 \pm 0.04$.

The fit can be improved by allowing for ionized reflection (model B). Assuming the reprocessor temperature $T=10^{6} \mathrm{~K}$ (for the purpose of computing ionization balance only), the best fit has $\chi^{2}=15.2 / 24$ d.o.f. for $\xi=0.1^{+4}$. If, instead, we let iron abundance be free whilst fixing $\xi=0$ (model C), we obtain $\chi^{2}=19.1 / 24$ d.o.f. for $[\mathrm{Fe}]=1.20 \pm 0.35$, i.e. $[\mathrm{Fe}]$ is not a significant parameter. The best fit with both $\xi$ and [Fe] free (model D) has $\chi^{2}=14.9 / 23$ d.o.f. for $\xi=0.1$ but again [Fe] is consistent with the cosmic value.

We now introduce the effect of smearing (model E; both $\xi$ and [Fe] are left free as well). This results in a decrease of $\chi^{2}$ by $\Delta \chi^{2}=8.9$ which is significant at more than $99.9 \%$ confidence level (the F-test for one additional parameter). The best fit inner radius is $R_{\text {in }}=25_{-11}^{+45} R_{\mathrm{g}}$. Figure 1 
shows the spectrum and residuals of the best fit models D and E.

A similar progression in quality of the fit is given by the July spectrum (Table 2; Figure 1), where again the relativistic smearing effects are significantly present in the data.

We have also tried a model with $\xi$ changing as a function of radius, $\xi(r) \propto r^{\alpha}$, but with no relativistic smearing. Best fit of the model for the June data has $\chi^{2}=14.6 / 22$ d.o.f., while for the July data $\chi^{2}=20.1 / 22$ d.o.f.

\section{Discussion}

\subsection{Primary continuum}

From fits we have $\Gamma \sim 1.7$, while contemporaneous high energy data show a cutoff at about 100 $\mathrm{keV}$ (Sunyaev et al. 1991). The source must then be marginally optically thin if the primary X-rays are produced by thermal Comptonization, with $\tau_{\mathrm{T}} \sim 0.5$ and 1.5, in a disk or sphere geometry, respectively (Titarchuk 1994).

\subsection{Geometry}

The results of spectral modelling clearly show that the reprocessed component is present in these data. The required smearing cannot be explained by a radial distribution of ionization since the data strongly favour a low and uniform ionization. Thus the relativistic effects in an accretion disk/black hole system are the most plausible explanation. The reflected fraction for both continuum and line is roughly $\sim 0.5 \times$ that expected from an isotropically illuminated flat disk. Thus the line is not depleted by Auger ionization masked by relativistic smearing (Ross et al. 1996). The line is weak because the covering fraction of the reflecting material is small, and Auger ionization is ruled out by the low ionization state of the disk. The low covering fraction does not seem to be an artifact of super-solar abundances: fixing $f=1$ and allowing the abundance to be free results in a poorer fit, with $\chi^{2}=19 / 23$ d.o.f. and $16 / 23$ d.o.f. for the June and July data, respectively, and the overall abundances, $A \sim 8$. It is also commonly seen in the persistent BHC: Cyg X-1 and GX 339-4 (Done et al. 1992; Gierliński et al. 1997; Ueda et al. 1994), so is not some time dependent effect of disk evolution in transient systems, but rather represents a significant geometrical constraint. Another constraint comes from the fact that the observed relativistic smearing is less than that expected from a central point source illuminating a flat disk which extends down to $6 R_{\mathrm{g}}$.

One possible geometry is a spherical or flattened X-ray source centered on the black hole as originally proposed by Thorne \& Price (1975) for Cyg X-1. A spherical source with $\tau_{\mathrm{T}} \gg 1$ gives $f=0.5$ naturally (Done et al. 1992), as the $\mathrm{X}$-ray emission only escapes into a hemisphere 
tangential to the source surface. However, here the source is probably marginally optically thin with $\tau_{T} \sim 0.5-2$ (see $\left.\S 5.1\right)$. This can still give a reduction in $f$ since there is a hole in the inner disk at $r \leq R_{\text {in }}$. Some of the photons escape without illuminating the disk, and the remaining reflection fraction is diluted by hard $\mathrm{X}$-ray photons produced on the other side of the disk. The relativistic smearing constraints then imply that the the source emissivity is less steep than $\propto r^{-3}$ and/or the inner disk disk truncates at $R_{\text {in }}>6 R_{\mathrm{g}}$ and/or the outer disk flares. There are few real physical constraints on the source emissivity. Local release of gravitational energy in a Keplerian disk gives a luminosity $\propto r^{-3}$, but non-local mechanisms could also operate, perhaps even producing a constant source $\propto r^{0}$. Outside of the source the illumination is approximated by a central point source i.e. $\propto r^{-3}$ but within the spherical source the illumination produces an emissivity of $\propto r^{-2}$ (for luminosity $\propto r^{-3}$ ). This can reduce the most strongly smeared components to the observed level, though truncation of the inner disk radius may also be required. The geometry might then be in accord with recently proposed scenario of SXT evolution after outbursts (Esin, McClintock \& Narayan 1997 and references therein) although the overall behavior of the source does not seem to follow the proposed scenario.

Small active regions on the disk (a "patchy" corona), perhaps powered by magnetic reconnection give another possible geometry (Haardt, Maraschi \& Ghisellini 1994, Stern et al. 1995). Physical support for such a picture may come from recent models of accretion disk viscosity as an MHD disk dynamo (Hawley, Gammie \& Balbus 1996). However, these models give $f \sim 1$ unless the height of the reconnecting regions, $h_{\text {rec }}$, is large compared to the size of the disk. This would require that the reconnecting regions be concentrated along the inner disk radius $R_{\text {in }}$, and that $h_{\text {rec }} \sim R_{\text {in }}$, since the outer disk is expected to be very large. This would also go some way to satisfying the smearing constraints, as the irradiation would then be constant from $\sim R_{\text {in }}$ to $\sim R_{\text {in }}+h_{\text {rec }}$ rather than $\propto r^{-3}$.

Another geometry that has been proposed, though with rather less physical motivation, is a continuous corona overlying the accretion disk (Haardt \& Maraschi 1993). A fraction $\sim 1-$ $\exp (-\tau / \cos i) \sim 0.5$ of the reflected spectrum is itself Compton scattered by the X-ray emitting corona, and loses its characteristic spectral shape (Haardt et al. 1993). The energy generation would be expected to be $\propto r^{-3}$, so the smearing constraints are a problem unless the inner disk truncates, or the outer disk flares. However, this simple scenario can be ruled out from detailed spectral fitting of Cyg X-1 (Gierliński et al. 1997; Poutanen, Krolik \& Ryde 1997), although perhaps this merely points to added complexity such as temperature/optical depth structure in the corona.

Additional independent constraints on the geometry are provided by low normalization of the soft component and weak ionization of the reflecting medium. They both support the idea of the disk being truncated at $\sim 30 R_{\mathrm{g}}$ as then both the expected temperature of Shakura-Sunyaev (1973) disk emission component (for $\dot{m}=0.01 M_{\text {Edd }}$ and $M=10 M_{\odot}$ ), $T \approx 0.15 \mathrm{keV}$ and the ionization parameter, $\xi \sim 1$, would be in agreement with our results. However, low $\xi$ may also mean that the disk is much denser than the SS solution due to e.g. coronal dissipation of energy (e.g. Svensson \& Zdziarski 1994). 


\section{Conclusions}

We have performed spectral analysis of Ginga data of soft X-ray transient GS 2023+338 (V404 Cyg) obtained one and two months after its 1989 outburst. We have found that

- the Compton reflected continuum and iron fluorescent $\mathrm{K} \alpha$ line are present in the spectrum,

- the properties of both reprocessed components (normalizations, ionization parameters) are in agreement,

- the data require both reprocessed components to be broadened and smeared,

- the smearing is consistent with being due to reflection from a Keplerian disk,

- both the normalization of the reflected continuum and the amount of smearing constrain the geometry.

This research made use of data obtained from the Leicester Database and Archive Service at the Department of Physics and Astronomy, Leicester University. CD acknowledges support from a PPARC Advanced Fellowship. Work of PTZ was partly supported by grant No. 2P03D00410 of the Polish State Committee for Scientific Research. 


\section{REFERENCES}

Done, C., Mulchaey, J. S., Mushotzky, R. F., \& Arnaud, K. A. 1992, ApJ, 395, 275

Esin, A. A., McClintock, J. E., \& Narayan, R. 1997, ApJ, submitted, astro-ph/9705237)

Fabian, A. C., Rees, M. J., Stella, L., \& White, N. E. 1989, MNRAS, 238, 729

Gierliński, M., Zdziarski, A. A., Done, C., Johnson, W. N., Ebisawa, K., Ueda, Y., \& Phlips, F. 1997, MNRAS, 288, 958

George, I. M., \& Fabian, A. C. 1991, MNRAS, 249, 352

Haardt, F., \& Maraschi, L. 1993, ApJ, 413, 507

Haardt, F., Maraschi, L., \& Ghisellini, G. 1994, ApJ, 432, L95

Haardt, F., Done, C., Matt, G., \& Fabian, A. C. 1993, ApJ, 411, L95

Hawley, J. F., Gammie, C. F., \& Balbus, S. A. 1996, ApJ, 464, 690

Inoue, H. 1993, in Accretion Disks in Compact Stellar Systems, ed. J. C. Wheeler (Singapore: World Scientific), 303

Iwasawa, K., et al. 1996, MNRAS, 282, 1038

Kaastra, J. S., \& Mewe, R. 1993, A\&AS, 97, 443

Kitamoto, S., Tsunemi, H., Miyamoto, S., Yamashita, K., \& Mizobuchi, S. 1989, Nature, 342, 518

Laor, A., 1991, ApJ, 376, 90

Lightman, A. P., \& White, T. R., 1988, ApJ, 335, 57

Magdziarz, P., \& Zdziarski, A. A. 1995, MNRAS, 273, 837

Matt, G., Perola, G. C., \& Piro, L. 1991, A\&A, 247, 25

Mitsuda, K., et al. 1984, PASJ, 36, 741

Morrison, R., \& McCammon, D. 1983, ApJ, 270119

Nandra, K., George, I.M., Mushotzky, R. F., Turner, T. J. \& Yaqoob, T. 1997, ApJ, 477, 602

Pavlenko, E. P., Martin, A. C., Casares, J., Charles, P. A., \& Ketsaris N. A. 1996, MNRAS, 281, 109

Poutanen, J., Krolik, J. H., \& Ryde, F. 1997, in Proceedings of 4th Compton Symposium, eds. C. D. Dermer and J. D. Kurfess, AIP, in press

Ross, R. R., Fabian, A. C., \& Brandt, W. N. 1996, MNRAS, 278, 1082

Shakura, N. I., \& Sunyaev, R. A. 1973, A\&A, 24, 337

Stern, B. E., Poutanen, J., Svensson, R., Sikora, M., \& Begelman, M. C. 1995, ApJ, 449, L13

Sunyaev, R. A., et al. 1991, Soviet Astron. Lett., 17, 123

Svensson, R., \& Zdziarski, A. A. 1994, ApJ, 436, 599 
Tanaka, Y., \& Lewin, W. H. G. 1995, in X-Ray Binaries, ed. W. H. G. Lewin, J. van Paradijs \& E. van den Heuvel (Cambridge: Cambridge Univ. Press), 126

Tanaka, Y., \& Shibazaki, N. 1996, ARA\&A, 34, 607

Tanaka, Y. et al. 1995, Nature, 375, 659

Thorne, K. S., \& Price, R. H. 1975, ApJ, 195, L101

Titarchuk, L. 1994, ApJ, 434, 570

Ueda, Y., Ebisawa, K., \& Done C. 1994, PASJ, 46, 107

Życki, P. T., \& Czerny, B. 1994, MNRAS, 266, 653 

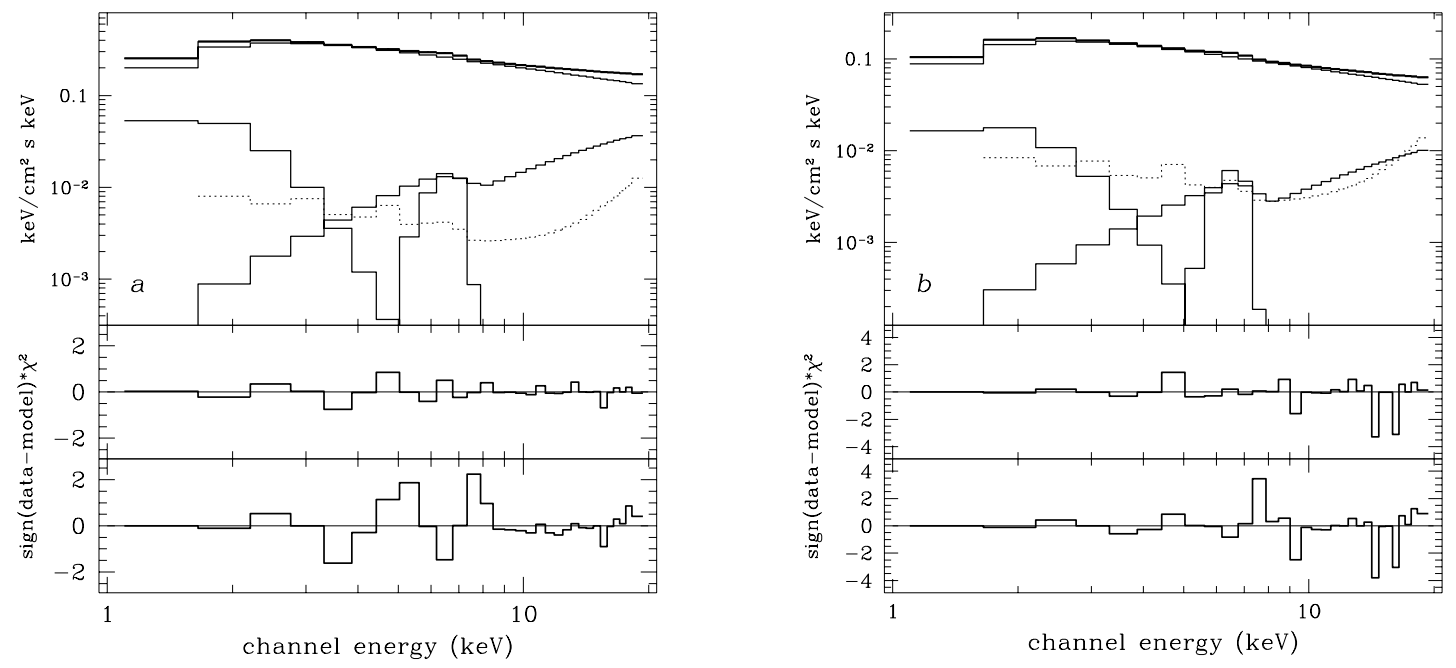

Fig. 1. - Best fit spectra (models E in Tables 1. and 2.; upper panels), their $\chi^{2}$ residuals (middle panels) and the $\chi^{2}$ residuals for models $\mathrm{D}$ i.e. without kinematic smearing. The dotted histograms show the background. Left panels (a) are for June data, right panels (b) for July data. 
Table 1. June 20th data

\begin{tabular}{ccccccccc}
\hline \hline model & $k T \mathrm{keV}$ & $\Gamma$ & $\xi$ & {$[\mathrm{Fe}]$} & $f$ & $R_{\text {in }}\left(R_{\mathrm{g}}\right)$ & $\mathrm{EW}(\mathrm{eV})$ & $\chi^{2} /$ d.o.f. \\
\hline 0 & $1.51 \pm 0.08$ & $1.34 \pm 0.02$ & $\ldots$ & $\ldots$ & $\ldots$ & $\ldots$ & $90_{-14}^{+23}$ & $46.8 / 25$ \\
$\mathrm{~A}$ & $0.36 \pm 0.04$ & $1.66 \pm 0.01$ & $0(\mathrm{f})$ & $1(\mathrm{f})$ & $0.46 \pm 0.04$ & $\ldots$ & $\ldots$ & $19.9 / 25$ \\
$\mathrm{~B}$ & $0.36_{-0.04}^{+0.09}$ & $1.66_{-0.03}^{+0.01}$ & $0.1^{+4}$ & $1(\mathrm{f})$ & $0.45_{-0.06}^{+0.04}$ & $\ldots$ & $\ldots$ & $15.2 / 24$ \\
$\mathrm{C}$ & $0.38_{-0.05}^{+0.17}$ & $1.64 \pm 0.04$ & $0(\mathrm{f})$ & $1.2 \pm 0.35$ & $0.43 \pm 0.06$ & $\ldots$ & $\ldots$ & $19.1 / 24$ \\
$\mathrm{D}$ & $0.37_{-0.05}^{+0.18}$ & $1.65 \pm 0.03$ & $0.1^{+4}$ & $1.0_{-0.2}^{+0.4}$ & $0.44 \pm 0.06$ & $\ldots$ & $\ldots$ & $14.9 / 23$ \\
$\mathrm{E}$ & $0.39_{-0.05}^{+0.25}$ & $1.64 \pm 0.04$ & $0^{+5}$ & $1.5_{-0.5}^{+0.7}$ & $0.47 \pm 0.06$ & $25_{-11}^{+45}$ & $\ldots$ & $6.0 / 22$ \\
\hline
\end{tabular}


Table 2. July data

\begin{tabular}{ccccccccc}
\hline \hline model & $k T \mathrm{keV}$ & $\Gamma$ & $\xi$ & {$[\mathrm{Fe}]^{\mathrm{a}}$} & $f$ & $R_{\text {in }}\left(R_{\mathrm{g}}\right)$ & $\mathrm{EW}(\mathrm{eV})$ & $\chi^{2} /$ d.o.f. \\
\hline 0 & $1.4 \pm 0.1$ & $1.45 \pm 0.25$ & $\ldots$ & $\ldots$ & $\ldots$ & $\ldots$ & $93 \pm 14$ & $57 / 25$ \\
$\mathrm{~A}$ & $0.36 \pm 0.03$ & $1.72 \pm 0.015$ & $0(\mathrm{f})$ & $1(\mathrm{f})$ & $0.40 \pm 0.05$ & $\ldots$ & $\ldots$ & $34.6 / 25$ \\
$\mathrm{~B}$ & $0.44_{-0.09}^{+0.40}$ & $1.68 \pm 0.04$ & $13_{-12}^{+25}$ & $1(\mathrm{f})$ & $0.32 \pm 0.08$ & $\ldots$ & $\ldots$ & $22.9 / 24$ \\
$\mathrm{C}$ & $0.42_{-0.06}^{+0.30}$ & $1.68 \pm 0.03$ & $0(\mathrm{f})$ & $1.7_{-0.5}^{+0.8}$ & $0.35 \pm 0.05$ & $\ldots$ & $\ldots$ & $26.9 / 24$ \\
$\mathrm{D}$ & $0.42_{-0.06}^{+0.50}$ & $1.68_{-0.06}^{+0.04}$ & $0.1^{+40}$ & $1.5 \pm 0.6$ & $0.35_{-0.10}^{+0.05}$ & $\ldots$ & $\ldots$ & $21.5 / 23$ \\
$\mathrm{E}$ & $0.47_{-0.09}^{+0.35}$ & $1.67_{-0.05}^{+0.04}$ & $0^{+20}$ & $2_{-0.8}^{+1}$ & $0.37_{-0.08}^{+0.05}$ & $35_{-21}^{+110}$ & $\ldots$ & $15.4 / 22$ \\
\hline
\end{tabular}

${ }^{\mathrm{a}}$ Upper limit on $[\mathrm{Fe}]$ is 3 